Article

\title{
Catalytic Asymmetric Fluorination of Alkyl 1-indanone-2-carboxylates Ruled by Pybox-Eu(III) Combination
}

\author{
Albert Granados $\mathbb{D}^{\text {, Pau Sarró and Adelina Vallribera * }}$ \\ Department of Chemistry, Universitat Autònoma de Barcelona and Centro de Innovación en Química \\ Avanzada (ORFEO-CINQA), Cerdanyola del Vallès, 08193 Barcelona, Spain; albert.granados@uab.es (A.G.); \\ psarro@iciq.es (P.S.) \\ * Correspondence: adelina.vallribera@uab.es; Tel.: +34-93-5813045
}

Received: 7 March 2019; Accepted: 20 March 2019; Published: 22 March 2019

\begin{abstract}
A highly enantioselective catalytic method for the synthesis of quaternary $\alpha$-fluoro derivatives of 3-oxo esters is described. The reaction uses europium (III) triflate and commercially available chiral pybox-type C2-symmetric ligand. Excellent results in terms of yields and enantioselectivities were assured using the electrophilic NFSI reagent under mild reaction conditions.
\end{abstract}

Keywords: europium; fluorination; enantioselectivity; pybox; keto ester

\section{Introduction}

Fluorine has drawn great interest from the scientific community. It has been used for a broad variety of purposes in all fields of chemistry, from the polymer industry, with hydrophobic plastics such as Teflon, to the nuclear power industry, or in the production of refrigerants and new solvents [1-4]. Of special interest is its use in pharmaceutical chemistry, where about $20 \%$ of all pharmaceuticals contain fluorinated moieties ( $30 \%$ in the case of agrochemicals) [5-13]. It is well known that the exchange of a hydrogen by a fluorine atom in a therapeutic molecule can produce a dramatic change on its physical and chemical properties, along with its biological activity. Generally, the addition of fluorine containing substituents in a drug enhances its metabolic stability and allows modulating its lipophilicity and bioavailability $[12,13]$. Thus, due to its importance in the pharmaceutical industry, the enantioselective synthesis of fluorinated molecules [14-16] is a great challenge in synthetic organic chemistry, especially under catalytic conditions.

Optically active $\alpha$-fluorinated cyclic $\beta$-keto esters are attractive compounds. On the one hand, they are regarded as non-enolizable and configurationally stable $\beta$-keto esters. Moreover, since the ketone is easily converted to other functional groups, they are versatile synthetic precursors. The development of stable sources of electrophilic fluorinating agents (Figure 1) such as $\mathrm{N}$-fluorobenzensulfonimide [17] (NFSI), Selectfluor ${ }^{\circledR}$ [18] and 1-fluoro-1,3-dihydro-3,3-dimethyl-1,2-benziodoxole, have had a great impact on catalytic enantioselective electrophilic fluorination. An important breakthrough in this chemistry occurred when Togni and Hintermann [19] described the catalytic $\alpha$-fluorination reactions of $\alpha$-substituted acyclic $\beta$-keto esters using a taddol-titanium complex and Selectfluor ${ }^{\circledR}$, yielding the fluorinated compounds in 33-90\% ee. Remarkable contributions of the group of Sodeoka [20-22] using palladium-BINAP chiral complexes; Togni [23,24] using Ru(II) chiral complexes; and Cahard [25] using $\mathrm{Cu}$ (II) bis(oxazoline) (Box) chiral complexes merit also to be mentioned. Much effort has been devoted to the $\alpha$-fluorination of alkyl 1-indanone-2-carboxylates under metal catalyst (Scheme 1). Sodeoka's group using a BINAP-Pd(II) complex in the reaction of tert-butyl 1-indanone-2-carboxylate and NFSI afforded the $\alpha$-fluorination product in $83 \%$ ee. [20] Then, Cahard and collaborators 
described [25] the reaction of benzyl 1-indanone-2-carboxylate and NFSI under (R)-Ph-Box-Cu(II) catalysis obtaining $35 \%$ ee ( 1 example). In 2004, Shibata's group performed a study using the combination of $\mathrm{Ni}\left(\mathrm{ClO}_{4}\right)_{2} \cdot 6 \mathrm{H}_{2} \mathrm{O}$ and $\mathrm{Ph}-\mathrm{Box}-\mathrm{Cu}(\mathrm{II})$ as catalyst and they succeeded with obtaining a $93 \%$ $e e$ in the reaction of tert-butyl 1-indanone-2-carboxylate and NFSI [26]. Moreover, using Nap-Box-Cu(II) and NFSI as fluorinating reagent, Kesavan's group obtained $34 \%$ ee in the $\alpha$-fluorination of ethyl 1-indanone-2-carboxylate [27]. The use of iron(III)-salan complexes, NFSI, and $\mathrm{AgClO}_{4}$ has also been described, affording a large scope of $\alpha$-fluorinated alkyl 1-indanone-2-carboxylates in good to excellent $e e$ values up to $97 \%$. [28] Moreover, $\mathrm{Xu}$ and collaborators [29] have reported a fast and highly enantioselective fluorination of different alkyl 1-indanone-2-carboxylates catalyzed by a chiral non commercial diphenylamineBox and $\mathrm{Cu}(\mathrm{OTf})_{2}$. The reactions were conducted using a ball mill apparatus [29] (Fritsch Planetary Micro Mill model "Pulverisette 7") in the absence of solvent and yielding the fluorinated compounds with enantioselectivities up to $99 \%$ ee (19 examples, $27-99 \%$ ee). However, the obtained ee's depended on the milling period and rotational speed, and normally in this kind of chemistry, contamination of products can occur as a result of wear and tear principally from the balls, and partially from the casing, which can be a problem, for example, in preparing building blocks for pharmaceuticals. To overcome these disadvantages, and due to our consolidated experience in the enantioselective $\alpha$-amination of $\beta$-keto esters catalyzed by the combination of lanthanides and pybox ligands [30-33], herein we report the results on catalytic enantioselective electrophilic $\alpha$-fluorination reaction of alkyl 1-indanone-2-carboxylates employing fully commercially available reagents.

\section{Pybox ligands}<smiles>c1ccc(C2COC(c3cccc(C4=NC(c5ccccc5)CO4)n3)=N2)cc1</smiles>

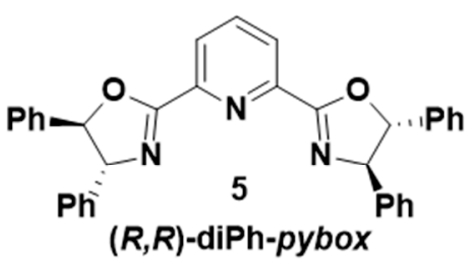<smiles>c1ccc(C2=NC(c3ccccc3)C(c3ccccc3)O2)cc1</smiles>

$(S, R)$-diPh-pybox

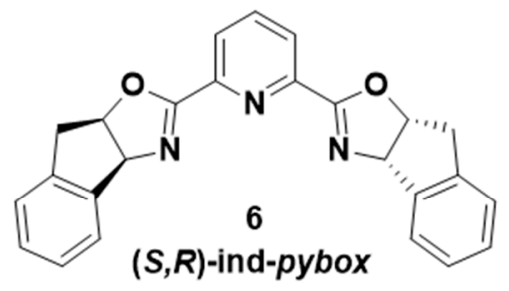

\section{Fluorinating electrophilic agents}<smiles>O=S(=O)(c1ccccc1)N(F)S(=O)(=O)c1ccccc1</smiles>

7

NFSI

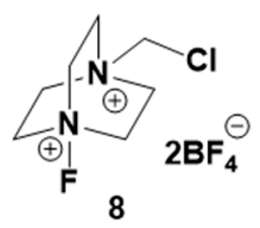

Selectfluor ${ }^{\circledR}$

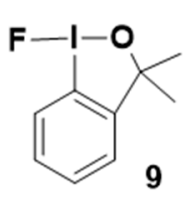

Figure 1. Fluorinating agents and pybox C2-symmetry ligands used in the optimization of the electrophilic enantioselective fluorination reaction. 
Previous work:

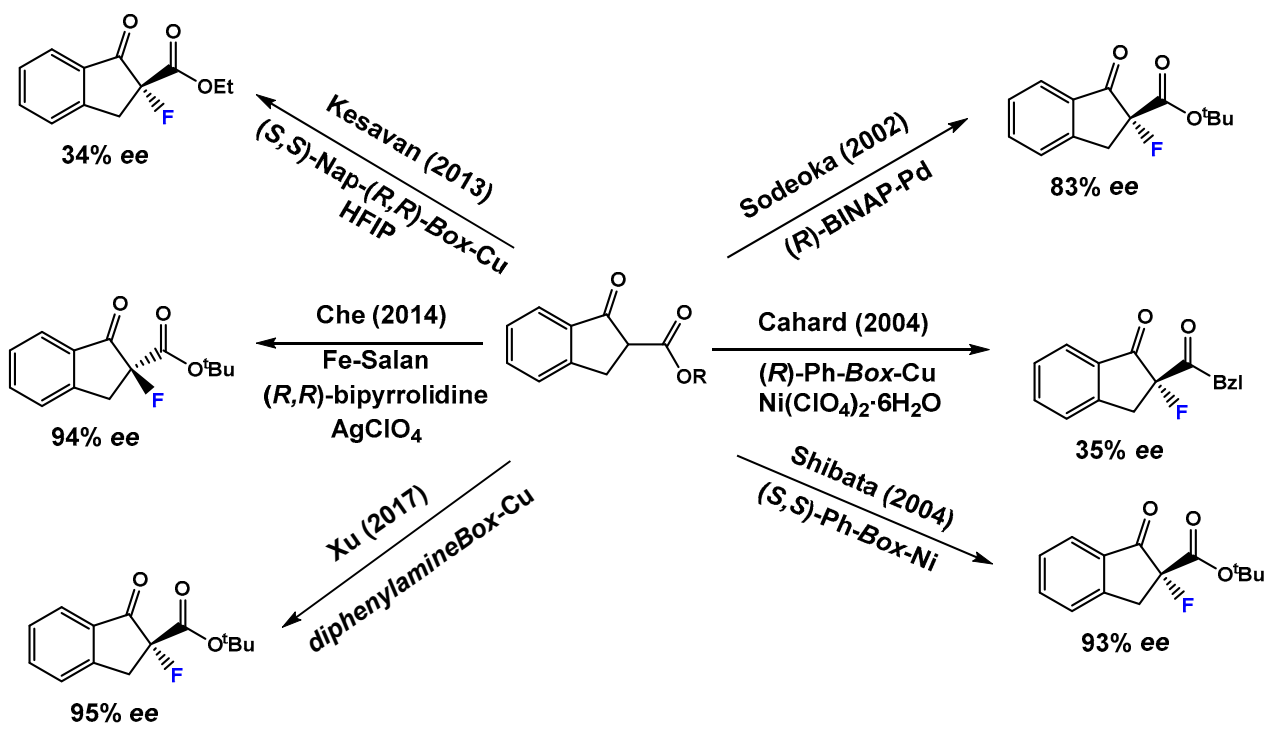

Scheme 1. Previous relevant work on enantioselective $\alpha$-fluorination of alkyl 1-indanone-2-carboxylates using NFSI under metal catalyst.

\section{Results and Discussion}

In the first part of this present work, we prepared a series of $\beta$-keto esters 2 derived from the corresponding 1-indanones. The acylation reaction of 1-indanones was carried out using sodium hydride as a base and dimethylcarbonate as electrophile in THF, obtaining compounds $\mathbf{1 a}-\mathbf{h}$ and $\mathbf{1} \mathbf{j}$ in excellent yields (Scheme 2, 75-100\%). In the case of $1 \mathbf{i}$ ( $87 \%$ yield), methyl $1 \mathrm{H}$-imidazole-1-carboxylate was used as acylating reagent; otherwise, exchange of the fluorine atom in 5 position by methoxide was observed [33]. Next, following the reported methodology of our research group [34], we performed the transesterification reaction to successfully afford the secondary and tertiary $\beta$-keto esters 2 shown in Scheme 2 (54-89\%).

Our previous experience suggested that the use of $\beta$-keto esters bearing a bulkier OR group might be useful to achieve enantioinduction, and secondary 3-pentyl group fulfills this requirement. Therefore, we selected $\mathbf{2 b}$ to investigate the effect of different combinations of lanthanides and pybox ligands in the $\alpha$-fluorination process. In the first attempt, we used europium (III) triflate and $(R, R)$-Ph-pybox (3) as catalyst and NFSI (7) as the fluorinating agent (Table 1, entry 1). Selection of NFSI was based in previous reported enantioselective fluorination reactions $[25,28]$. Under these conditions, product $10 \mathrm{~b}$ was afforded in a $76 \%$ yield but a negligible enantiomeric ratio. Next, we changed the metal source, moving to lanthanum and ytterbium and obtaining in both cases $10 \mathrm{~b}$ in nearly racemic form. At this point, we decided to change the chiral ligand. From our previous experience [31,32], we envisioned good results using more sterically hindered pybox ligands 4 and 5. They are non-commercially available and were prepared as previously described [31,35]. The ee's improved but not enough (30-56 \% ee, entries $4-7$ of Table 1). Then, we studied the commercially available $(S, R)$-ind-pybox, 6, and the results improved in a great extend, achieving the highest yield (87\%) in combination with $\mathrm{La}(\mathrm{III})$ and affording enhanced enantioselective ratios specially in the case of $\mathrm{Eu}(\mathrm{III})\left(78 \%\right.$ ee) (entries $8-10$ of Table 1). Selectfluor ${ }^{\circledR}$ (8) and 1-fluoro-1,3-dihydro-3,3-dimethyl-1,2-benziodoxole (9) were assayed giving worse results in terms of yield and $e e$ (entries 11 and 12, Table 1). The hypervalent iodine fluorinated reagent 9 produces the oxidation of the $\alpha$-position, affording the corresponding $\alpha$-hydroxylated $\beta$-keto ester as the only detected product. This oxidation process has previously been described in the electrophilic cyanation of $\beta$-keto esters using 1-cyano-1,3-dihydro-3,3-dimethyl-1,2-benziodoxole [36]. So, we proceed again with the optimized reaction, using NFSI, but now at a low temperature $\left(-30^{\circ} \mathrm{C}\right)$. The reaction of $\mathbf{2 b}$ 
and 1a catalyzed by ind-pybox-La(III) gave rise to the fluorinating products in good yields ( 60 and $80 \%$ respectively) and moderate $e e^{\prime} s$ (64 and $63 \%$ respectively). Additionally, performing the reaction of $2 \mathrm{c}$ (tert-butyl ester) in the same conditions, product 10c was obtained in an enhanced $76 \%$ ee (entry 15 , Table 1). Finally, changing to ind-pybox-Eu(III) compound 10c was achieved in an excellent $96 \% e e$ (entry 16, Table 1).

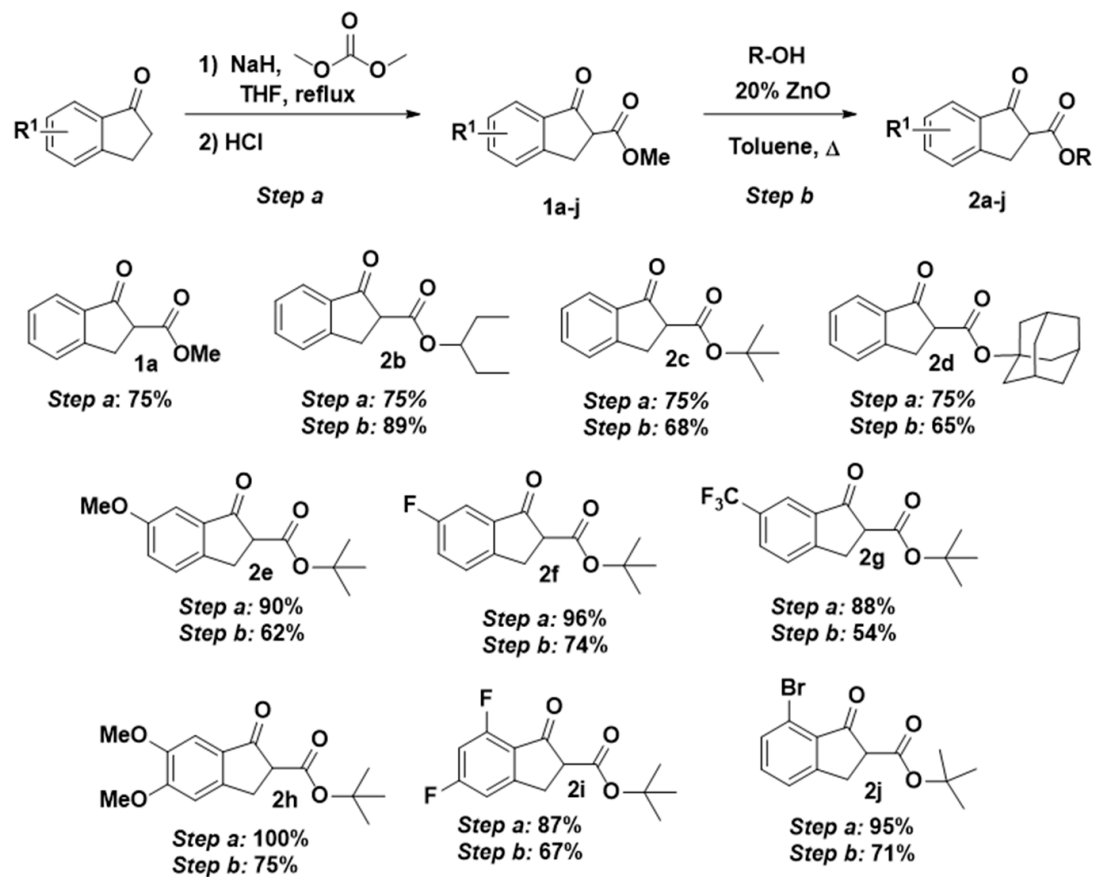

Scheme 2. Preparation of alkyl 1-indanone-2-carboxylates 1 and 2.

Table 1. Optimization of the enantioselective $\alpha$-fluorination reaction.

\begin{tabular}{|c|c|c|c|c|c|c|}
\hline \multirow[b]{2}{*}{ Entry } & \multirow[b]{2}{*}{2} & \multirow[b]{2}{*}{ Metal } & \multicolumn{2}{|c|}{$\begin{array}{l}\begin{array}{c}\text { Fluorinanting Reagent } \\
\mathrm{M}(\mathrm{OTf})_{3}, \text { pybox }\end{array} \\
\underset{\mathrm{ACN}, \mathrm{rt}, 4 \mathrm{~A} \text { MS }}{\longrightarrow}\end{array}$} & \multirow[b]{2}{*}{ Yield (\%) } & \multirow[b]{2}{*}{$e e(\%)[a]$} \\
\hline & & & Ligand & Fluorinating Reagent & & \\
\hline 1 & $2 b$ & $\mathrm{Eu}^{+3}$ & 3 & NFSI & 76 & 2 \\
\hline 2 & $2 b$ & $\mathrm{La}^{+3}$ & 3 & NFSI & 74 & 5 \\
\hline 3 & $2 b$ & $\mathrm{Yb}^{+3}$ & 3 & NFSI & 68 & 2 \\
\hline 4 & $2 b$ & $\mathrm{La}^{+3}$ & 4 & NFSI & 81 & 56 \\
\hline 5 & $2 b$ & $\mathrm{Eu}^{+3}$ & 4 & NFSI & 45 & 30 \\
\hline 6 & $2 b$ & $\mathrm{Yb}^{+3}$ & 4 & NFSI & 78 & 54 \\
\hline 7 & $2 b$ & $\mathrm{La}^{+3}$ & 5 & NFSI & 85 & 50 \\
\hline 8 & $2 b$ & $\mathrm{La}^{+3}$ & 6 & NFSI & 87 & 60 \\
\hline 9 & $2 b$ & $\mathrm{Eu}^{+3}$ & 6 & NFSI & 72 & 78 \\
\hline 10 & $2 b$ & $\mathrm{Yb}^{+3}$ & 6 & NFSI & 70 & 60 \\
\hline 11 & $2 b$ & $\mathrm{La}^{+3}$ & 6 & 8 & 59 & 14 \\
\hline 12 & $2 b$ & $\mathrm{La}^{+3}$ & 6 & 9 & 0 & n.d. \\
\hline $13[\mathrm{~b}]$ & $1 b$ & $\mathrm{La}^{+3}$ & 6 & NFSI & 60 & 64 \\
\hline $14[\mathrm{~b}]$ & $1 a$ & $\mathrm{La}^{+3}$ & 6 & NFSI & 80 & 63 \\
\hline $15[\mathrm{~b}]$ & $2 c$ & $\mathrm{La}^{+3}$ & 6 & NFSI & 86 & 76 \\
\hline $16[\mathrm{~b}]$ & $2 c$ & $\mathrm{Eu}^{+3}$ & 6 & NFSI & 78 & 96 \\
\hline
\end{tabular}

[a] The racemic compounds 10 were prepared by reaction of the alkyl 1-indanone-2-carboxylates with Selectfluor ${ }^{\circledR}$ (1 equiv.) in $\mathrm{CH}_{3} \mathrm{CN}$ at $50^{\circ} \mathrm{C}$. The $e e^{\prime}$ s were determined by using chiral HPLC (see supplementary information). [b] Reaction done at $-30^{\circ} \mathrm{C}$. 
Therefore, as the best performance in terms of selectivity was obtained using sterically hindered tert-butyl 1-oxo-indanecarboxylate 2c (entry 16, Table 1), we also performed the reaction with adamantan-1-yl 1-oxo-2-indanecarboxylate in the same conditions, obtaining $10 \mathrm{~d}$ in a slightly lower 90\% ee (Figure 2). Thus, we focused our attention on other tert-butyl 1-indanone-2-carboxylates possessing different groups in the aromatic ring. $\alpha$-Fluorinated compounds $\mathbf{1 0 e}-\mathbf{j}$ were achieved in high yields (66-85\%) and enantiomeric excesses up to $93 \%$ when using ind-pybox-Eu(III) combination. Lanthanium gave lower selectivities in all the studied substrates (Figure 2). First, we selected three different substituents in 6 positions of the aromatic ring. The change from $\mathrm{OMe}(\mathbf{1 0 e}, 90 \% e e)$ to $\mathrm{CF}_{3}$ $(\mathbf{1 0 g}, 81 \% e e)$ lessen the $e e$ and the reaction time from $24 \mathrm{~h}$ to $8 \mathrm{~h}$. Addition of an extra OMe group in 5 position (10h) did not improve the ee (91\%). Compounds $\mathbf{1 0 i}$ and $\mathbf{1 0 j}$ were obtained in 84 and $85 \%$ ee respectively. In general, the presence of electron withdrawing groups harmed the $e e(\mathbf{1 0}, \mathbf{g}, \mathbf{i}$ and $\mathbf{1 0} \mathbf{j}$, Figure 2), except in the case of $\mathbf{1 0 f}$ (F in 6 position), which was obtained in an excellent $93 \%$ ee.
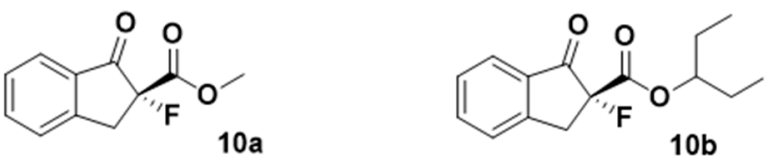

La (III) $80 \%$ yield, $62 \%$ ee

La (III) $60 \%$ yield, $64 \%$ ee<smiles>CC(C)(C)OC(=O)[C@]1(F)Cc2ccccc2C1=O</smiles>

La (III) $86 \%$ yield, $76 \%$ ee Eu (III) $78 \%$ yield, $96 \%$ ee<smiles>CC(C)(C)OC(=O)[C@]1(F)Cc2ccc(F)cc2C1=O</smiles>

La (III): $76 \%$ yield, $60 \%$ ee Eu(III): $71 \%$ yield, $93 \%$ ee<smiles>CC(C)(C)OC(=O)[C@]1(F)Cc2cc(F)cc(F)c2C1=O</smiles>

Eu(III): $71 \%$ yield, $84 \%$ ee<smiles>O=C(OC12CC3CC(CC(C3)C1)C2)[C@@]1(F)Cc2ccccc2C1=O</smiles>

La (III): $95 \%$ yield, $66 \%$ ee Eu (III): $85 \%$ yield, $90 \%$ ee<smiles>CC(C)(C)OC(=O)[C@]1(F)Cc2ccc(C(F)(F)F)cc2C1=O</smiles>

La (III): $\mathbf{8 2} \%$ yield, $\mathbf{5 0 \%}$ ee Eu (III): $80 \%$ yield, $81 \%$ ee<smiles>CC(C)(C)OC(=O)[C@]1(F)Cc2cccc(Br)c2C1=O</smiles>

Eu(III): $73 \%$ yield, $85 \%$ ee<smiles>COc1ccc2c(c1)C(=O)[C@](F)(C(=O)OC(C)(C)C)C2</smiles>

La (III): $66 \%$ yield, $72 \%$ ee Eu (III): $72 \%$ yield, $90 \%$ ee<smiles>COc1cc2c(cc1OC)C(=O)[C@](F)(C(=O)OC(C)(C)C)C2</smiles>

La(III) $: 72 \%$ yield, $74 \%$ ee Eu (III): $66 \%$ yield, $91 \%$ ee

Figure 2. Scope of the reaction of alkyl 1-indanone-2-carboxylates 2 with different groups in aromatic positions. All the reactions were carried out at the same conditions $\left(-30^{\circ} \mathrm{C}\right)$, see experimental part.

The assignment of the absolute configuration of compounds $\mathbf{1 0}$ was done by comparison of the sign of the optical rotation values already described for $\mathbf{1 0 c}-\mathbf{f}$ [28]. For new compounds $\mathbf{1 0 b}$ and $\mathbf{1 0 g}-\mathbf{j}$ the assignment was based on the comparison of the circular dichroism (CD) (all studied compounds show a negative Cotton Effect, see supplementary information). In all cases, the absolute configuration was assigned to be $R$. The advantage of this method is that we could also have access to (S)-10 enantiomers by using commercially available $(R, S)$-ind-pybox.

Furthermore, a plausible catalytic cycle is proposed for this enantioselective fluorination reaction (Scheme 3) $[30,33]$. First, we propose the formation of the $\left[p y b o x \operatorname{Eu}(\mathrm{OTf})_{2}\right]^{+}(\mathbf{a} 1)$ complex that can exchange the labile triflate ligand by the enolate form of the $\beta$-keto ester affording the complex [pybox $\mathrm{Eu}(\mathrm{OTf})(\text { enolate-2] }]^{+}$(a2). Similar complexes were detected by ESI Mass Spectometry when mixing $\mathrm{La}(\mathrm{OTf})_{3}$ and ip-pybox [30]. The pybox ligand is the responsible base for the formation of the 
enolate-2. Abstraction of the intercarbonylic proton can be produced directly from 2 (as indicated in Scheme 3); or once the $\beta$-keto ester is coordinated to the europium enhancing its acidity. Next, a coordination of the NFSI, through the donor oxygen atom, to the europium forming a ternary complex is proposed. The known large coordination number of lanthanides makes that possible. The enantioselectivity of the reaction stems from the efficient blockage of one of the diastereotopic faces of the $\mathrm{Eu}(\mathrm{III})$ enolate by one unit of the C2-symmetric ligand. Subsequent reaction affords the formation of the stereogenic $\mathrm{C}-\mathrm{F}$ bond through a $\mathrm{S}_{\mathrm{N}} 2$ mechanism.

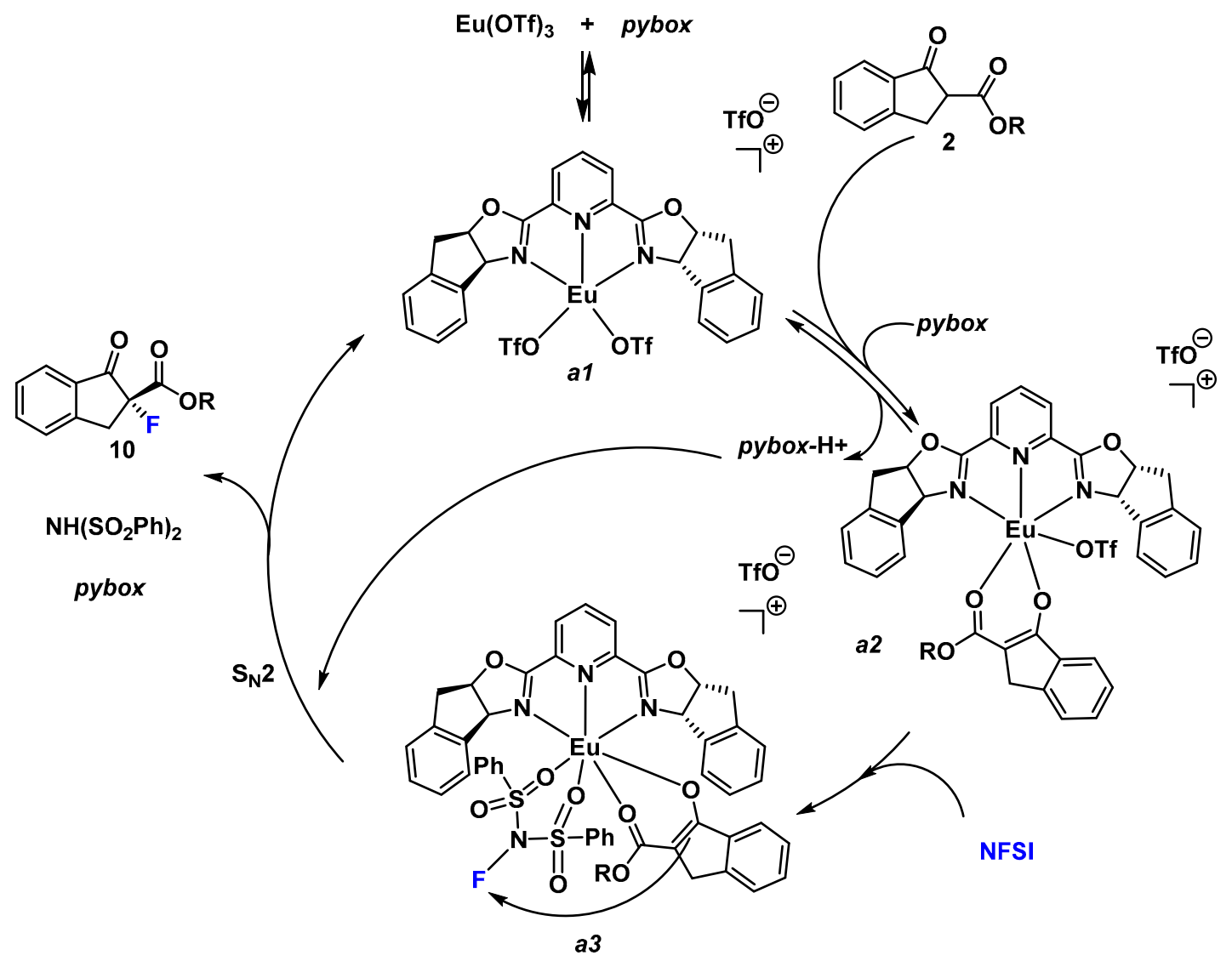

Scheme 3. Plausible catalytic cycle.

\section{Materials and Methods}

General transesterification procedure: In a round-bottomed flask, the corresponding 1-indanone (1 eq.), $\mathrm{ZnO}$ (0.2 eq.) and the corresponding alcohol (10 equivalents) were dissolved in toluene ( $5 \mathrm{~mL} / \mathrm{mol} 1$-indanone). The reaction mixture was heated up to $140{ }^{\circ} \mathrm{C}$ under a distillation setup until total conversion of the 1-indanone was observed by TLC (from 6 to $8 \mathrm{~h}$ ). If necessary, more toluene was added. Afterwards, the reaction mixture was filtered through Celite ${ }^{\circledR}$ and the solvent was removed under reduced pressure. The product obtained was purified by column chromatography on silica-gel.

Pentan-3-yl 1-oxo-2,3-dihydro-1H-indene-2-carboxylate (2b): Following the general procedure, $1.00 \mathrm{~g}$ (5.26 mmol, $1 \mathrm{eq})$ of 1a was allowed to react with 3-pentanol (52.7 mmol, 10 eq.) and $\mathrm{ZnO}$ (0.09 g, $1.06 \mathrm{mmol}, 0.2$ eq.) in $6 \mathrm{~mL}$ of toluene for $5 \mathrm{~h}$. After purification by column chromatography on silica-.gel (hexanes (95): AcOEt (5)) a brown oil was obtained in 90\% yield (1.17 g). IR (ATR) $v=$ 2968, 1711 (C=O), 1608, 1572, 1207, $1092 \mathrm{~cm}^{-1} ;{ }^{1} \mathrm{H}-\mathrm{NMR}(360 \mathrm{MHz}$ [D]CDCl $3,298 \mathrm{~K}, \mathrm{TMS}) \delta=10.50$ (bs, 0.08H, OH, enol), $7.79\left(\mathrm{~d},{ }^{3} J_{(H, H)}=7.5 \mathrm{~Hz}, 1 \mathrm{H}, \mathrm{ArH}\right), 7.64\left(\mathrm{t},{ }^{3} J_{(H, H)}=7.1 \mathrm{~Hz}, 1 \mathrm{H}, \mathrm{ArH}\right), 7.52(\mathrm{~d}$, $\left.{ }^{3} J_{(H, H)}=7.5 \mathrm{~Hz}, 1 \mathrm{H}, \mathrm{ArH}\right), 7.50-7.44\left(\mathrm{~m}, 0.17 \mathrm{H}, \mathrm{ArH}\right.$, enol), $7.41\left({ }^{3} J_{(H, H)}, J=7.5 \mathrm{~Hz}, 1 \mathrm{H}, \mathrm{ArH}\right), 4.98$ (quint, ${ }^{3} J_{(H, H)}=6.1 \mathrm{~Hz}, 0.16 \mathrm{H}, \mathrm{OCH}$, enol), 4.86 (quint, $\left.{ }^{3} J_{(H, H)}=6.3 \mathrm{~Hz}, 1 \mathrm{H}, \mathrm{OCH},\right), 3.74\left(\mathrm{dd},{ }^{3} J_{(H, H)}=4.2 \mathrm{~Hz}\right.$, $\left.{ }^{3} J_{(H, H)}=8.7 \mathrm{~Hz}, 1 \mathrm{H}, \mathrm{CH}_{2} \mathrm{CH}\right), 3.59\left(\mathrm{~d},{ }^{3} J_{(H, H)}=4.2 \mathrm{~Hz}, 0.42 \mathrm{H}, \mathrm{CH} \mathrm{C}\right.$, enol), $3.55\left(\mathrm{~d},{ }^{3} J_{(H, H)}=3.7 \mathrm{~Hz}\right.$, 
$\left.1 \mathrm{H}, \mathrm{CH}_{2} \mathrm{CH}\right), 3.40\left(\mathrm{dd},{ }^{3} \mathrm{~J}_{(\mathrm{H}, \mathrm{H})}=8.3 \mathrm{~Hz}, \mathrm{~J}=17.5 \mathrm{~Hz}, 1 \mathrm{H}, \mathrm{CH}_{2} \mathrm{CH}\right), 1.70\left(\mathrm{~m}, 1 \mathrm{H}, \mathrm{CH}_{2} \mathrm{CH}_{3}\right.$, enol), 1.65 $\left(\mathrm{m}, 4 \mathrm{H}, \mathrm{CH}_{2} \mathrm{CH}_{3}\right), 1.02-0.86$ ppm $\left(\mathrm{m}, 7.36, \mathrm{CH}_{2} \mathrm{CH}_{3}\right) ;{ }^{13} \mathrm{C}-\mathrm{NMR}\left(101 \mathrm{MHz},[\mathrm{D}] \mathrm{CDCl}_{3}, 298 \mathrm{~K}, \mathrm{TMS}\right) \delta$ $=199.6,169.0,153.6,143.1,137.0,135.3,135.2,129.1,127.7,126.7,126.5,124.6,124.6,120.6,102.7,78.3$, 76.4, 53.6, 32.52, 30.3, 26.6, 26.4, 9.6, 9.4 ppm; HRMS (ESI): $(\mathrm{M}+\mathrm{Na})^{+}$Calcd for $\mathrm{C}_{15} \mathrm{H}_{18} \mathrm{O}_{3} \mathrm{Na} 269.1148$; found 269.1144 .

Tert-butyl-5,7-difluoro-1-oxo-2,3-dihydro-1H-indene-2-carboxylate (2i): Following the general procedure, $0.50 \mathrm{~g}(2.21 \mathrm{mmol}, 1 \mathrm{eq})$ of $\mathbf{1 i}$ was allowed to react with tert-butanol (22.1 mmol, $10 \mathrm{eq}$.) and $\mathrm{ZnO}(0.04 \mathrm{~g}, 0.45 \mathrm{mmol}, 0.2$ eq.) in $5 \mathrm{~mL}$ of toluene for $5 \mathrm{~h}$. After purification by column chromatography on silica-.gel (hexanes (95): AcOEt (5)) a purple oil was obtained in 64\% yield (0.38 g). IR (ATR) $v=2961,1758(\mathrm{C}=\mathrm{O}), 1722,1617,1283,1045 \mathrm{~cm}^{-1},{ }^{1} \mathrm{H}-\mathrm{NMR}\left(360 \mathrm{MHz}[\mathrm{D}] \mathrm{CDCl}_{3}, 298 \mathrm{~K}\right.$ TMS) $\delta=10.62\left(\mathrm{bs}, 0.07 \mathrm{H}, \mathrm{OH}\right.$, enol), $6.97\left(\mathrm{~d},{ }^{3} J_{(H, F)}=7.3 \mathrm{~Hz}, 1 \mathrm{H}, \mathrm{ArH}\right), 6.74\left(\mathrm{t},{ }^{3} J_{(H, F)}=9.1 \mathrm{~Hz}, 1 \mathrm{H}\right.$, $\operatorname{ArH}), 3.64\left(\mathrm{dd},{ }^{3} J_{(H, H)}=3.9 \mathrm{~Hz},{ }^{3} J_{(H, H)}=8.2 \mathrm{~Hz}, 1 \mathrm{H}, \mathrm{CH}_{2} \mathrm{CH}\right), 3.48\left(\mathrm{dd},{ }^{3} J_{(H, H)}=4.4 \mathrm{~Hz},{ }^{3} J_{(H, H)}=16.7\right.$ $\left.\mathrm{Hz}, \mathrm{CH}_{2} \mathrm{CH}\right), 3.30\left(\mathrm{dd},{ }^{3} J_{(\mathrm{H}, \mathrm{H})}=8.3 \mathrm{~Hz}, J=17.6 \mathrm{~Hz}, 1 \mathrm{H}, \mathrm{CH}_{2} \mathrm{CH}\right), 1.47\left(\mathrm{~s}, 9 \mathrm{H}, \mathrm{C}\left(\mathrm{CH}_{3}\right)_{3}\right) ;{ }^{19} \mathrm{~F}-\mathrm{NMR}$ $\left(235 \mathrm{MHz}[\mathrm{D}] \mathrm{CDCl}_{3}, 298 \mathrm{~K}, \mathrm{TMS}\right) \delta=-99.9\left(\mathrm{~d},{ }^{3} J_{(F, F)}=13.7 \mathrm{~Hz}, 1 \mathrm{~F}\right),-111.3 \mathrm{ppm}\left(\mathrm{d},{ }^{3} J_{(F, F)}=13.7 \mathrm{~Hz}\right.$, $1 \mathrm{~F}) ;{ }^{13} \mathrm{C}-\mathrm{NMR}\left(91 \mathrm{MHz},[\mathrm{D}] \mathrm{CDCl}_{3}, 298 \mathrm{~K}, \mathrm{TMS}\right) \delta=194.5\left(\mathrm{~d},{ }^{3} J_{(C, F)}=2.2 \mathrm{~Hz}\right), 167.8\left(\mathrm{dd},{ }^{3} J_{(C, F)}=3.9 \mathrm{~Hz}\right.$, $\left.{ }^{1} J_{(C, F)}=259.5 \mathrm{~Hz}\right), 167.5(\mathrm{~s}), 160.0\left(\mathrm{dd},{ }^{3} J_{(C, F)}=14.2 \mathrm{~Hz},{ }^{1} J_{(C, F)}=267.5 \mathrm{~Hz}\right), 157.7(\mathrm{~m}), 120.1(\mathrm{~m}), 109.5$ $\left(\mathrm{dd}^{4} J_{(C, F)}=4.2 \mathrm{~Hz},{ }^{2} J_{(C, F)}=22.5 \mathrm{~Hz}\right), 103.9\left(\mathrm{dd},{ }^{2} J_{(C, F)}=22.7 \mathrm{~Hz},{ }^{2} J_{(C, F)}=24.7 \mathrm{~Hz}\right), 82.5(\mathrm{~s}), 55.0(\mathrm{~s}), 27.8$ (s) ppm; HRMS (ESI): (M + Na) Calcd for $\mathrm{C}_{14} \mathrm{H}_{14} \mathrm{~F}_{2} \mathrm{O}_{3} \mathrm{Na} 291.0803$; found 291.0805 .

General procedure for the enantioselective electrophilic fluorinations: In a $10 \mathrm{~mL}$ Schlenk flask in presence of $4 \AA \mathrm{MS}, \mathrm{Ln}(\mathrm{OTf})_{3}$ (0.13 eq.) and the desired pybox ligand (0.15 eq.) were dissolved with dry ACN $(5 \mathrm{~mL})$. The colorless reaction mixture was left stirring at room temperature under argon atmosphere overnight. Next, the corresponding indanone derivative 2 ( $80 \mathrm{mg} ; 1$ eq.) was added to the reaction mixture and left stirring at room temperature for $1 \mathrm{~h}$. Then, the reaction mixture was cooled down until $-30{ }^{\circ} \mathrm{C}$ and, once at this temperature, NFSI (1.15 eq.) was added to the mixture in one portion. The reaction mixture was left at this temperature under argon atmosphere until complete conversion of the reagent. Afterwards, the solvent was removed under reduced pressure and the product was purified by column chromatography on silica gel, yielding the $\alpha$-fluorinated compound.

Pentan-3-yl (R)-2-fluoro-1-oxo-2,3-dihydro-1H-indene-2-carboxylate (10b): Following the general procedure using $\mathrm{La}(\mathrm{OTf})_{3}$ and $(S, R)$-ind-pybox, $79 \mathrm{mg}(0.29 \mathrm{mmol}, 60 \%$ yield $)$ of $\mathbf{1 0 b}$ were obtained as a tan oil from $0.37 \mathrm{mmol}$ of $\mathbf{2 b}$. It was purified by column chromatography on silica-gel (hexane/ AcOEt 5:1). IR (ATR) $v=2970,1760(\mathrm{C}=\mathrm{O}), 1607(\mathrm{C}=\mathrm{O}), 1464,1287,1214,1191,1156,1100 \mathrm{~cm}^{-1} ;{ }^{1} \mathrm{H}-\mathrm{NMR}$ $\left(250 \mathrm{MHz}[\mathrm{D}] \mathrm{CDCl}_{3}, 298 \mathrm{~K}, \mathrm{TMS}\right) \delta=7.85\left(\mathrm{~d},{ }^{3} J_{(H, H)}=8.1 \mathrm{~Hz}, 1 \mathrm{H}, \mathrm{ArH}\right), 7.71\left(\mathrm{t},{ }^{3} J_{(H, H)}=7.4 \mathrm{~Hz}\right.$, $1 \mathrm{H}, \mathrm{ArH}), 7.57(\mathrm{~m}, 2 \mathrm{H}, \mathrm{ArH}), 4.90$ (quint, $\left.{ }^{3} J_{(H, H)}=6.3 \mathrm{~Hz}, 1 \mathrm{H}, \mathrm{OCH}\right), 3.78\left(\mathrm{dd},{ }^{2} J_{(H, H)}=11.4 \mathrm{~Hz}\right.$, $\left.{ }^{3} J_{(H, F)}=17.8 \mathrm{~Hz}, 1 \mathrm{H}, \mathrm{CH}_{2} \mathrm{CF}\right), 3.40\left(\mathrm{dd},{ }^{2} J_{(H, H)}=22.8 \mathrm{~Hz},{ }^{3} J_{(H, F)}=25.7 \mathrm{~Hz}, 1 \mathrm{H}, \mathrm{CH}{ }_{2} \mathrm{CF}\right), 1.55(\mathrm{~m}, 4 \mathrm{H}$, $\left.\mathrm{CH}_{2} \mathrm{CH}_{3}\right), 0.84(\mathrm{~m}, 6 \mathrm{H}) ;{ }^{13} \mathrm{C}-\mathrm{NMR}\left(91 \mathrm{MHz}[\mathrm{D}] \mathrm{CDCl}_{3}, 298 \mathrm{~K}, \mathrm{TMS}\right) \delta=195.5\left(\mathrm{~d},{ }^{2} J_{(\mathrm{C}, F)}=18.2 \mathrm{~Hz}\right), 167.1$ $\left(\mathrm{d},{ }^{2} J_{(C, F)}=27.8 \mathrm{~Hz}\right), 150.9\left(\mathrm{~d},{ }^{3} J_{(H, F)}=3.7 \mathrm{~Hz}\right), 136.4(\mathrm{~d}, J=3.0 \mathrm{~Hz}, \mathrm{Ar}-\mathrm{C}), 133.4(\mathrm{~s}), 128.6(\mathrm{~s}), 126.5$ $\left(\mathrm{d},{ }^{4} J_{(C, F)}=1.2 \mathrm{~Hz}\right), 125.5,94.5\left(\mathrm{~d},{ }^{1} J_{(C, F)}=201.2 \mathrm{~Hz}\right), 79.6(\mathrm{~s}), 38.4\left(\mathrm{~d},{ }^{2} J_{(C, F)}=24.1 \mathrm{~Hz}\right), 26.4(\mathrm{~s}), 26.3$ (s), 9.5 (s), 9.3 (s). ${ }^{19} \mathrm{~F}-\mathrm{NMR}(235 \mathrm{MHz}$ [D]CDCl $, 298 \mathrm{~K}, \mathrm{TMS}) \delta=-164.8 \mathrm{ppm}$ (s, 1F); HPLC: Daicel Chiralpack AD-H, Hexane $/{ }^{i} \mathrm{PrOH}=99.5: 0.5,0.75 \mathrm{~mL} / \mathrm{min}, 254 \mathrm{~nm}, \mathrm{t}_{r}$ (minor) = $33.4 \mathrm{~min}, \mathrm{t}_{r}($ major $)$ $=40.7 \min (64 \%$ ee $) ;[\alpha]_{20} \mathrm{D}:-10.3\left(\mathrm{c}=5.9, \mathrm{CHCl}_{3}\right)$. HRMS (ESI): $(\mathrm{M}+\mathrm{Na})^{+}$Calcd for $\mathrm{C}_{15} \mathrm{H}_{17} \mathrm{O}_{3} \mathrm{FNa}$ 287.1054; found 287.1059.

Tert-butyl (R)-2-fluoro-1-oxo-6-(trifluoromethyl)-2,3-dihydro-1H-indene-2-carboxylate (10g): Following the general procedure, using $\mathrm{Eu}(\mathrm{OTf})_{3}$ and $(S, R)$-ind-pybox, $67 \mathrm{mg}(0.21 \mathrm{mmol}, 80 \%$ yield $)$ of $10 \mathrm{~g}$ were obtained as a brown oil from $0.31 \mathrm{mmol}$ of the starting material. It was purified by column chromatography on silica-gel (hexane/AcOEt 7:3). IR (ATR) $v=2916,2849,1736$ (C=O), 1467, 1330, 1259, $1179 \mathrm{~cm}^{-1},{ }^{1} \mathrm{H}-\mathrm{NMR}\left(360 \mathrm{MHz}[\mathrm{D}] \mathrm{CDCl}_{3}, 298 \mathrm{~K}, \mathrm{TMS}\right) \delta=8.11(\mathrm{~s}, 1 \mathrm{H}, \mathrm{ArH}), 7.95\left(\mathrm{~d},{ }^{3} J_{(H, H)}=7.9 \mathrm{~Hz}\right.$, $1 \mathrm{H}, \mathrm{ArH}), 7.68\left(\mathrm{~d},{ }^{3} J_{(H, H)}=7.9 \mathrm{~Hz}, 1 \mathrm{H}, \mathrm{ArH}\right), 3.82\left(\mathrm{dd},{ }^{2} J_{(H, H)}=10.7 \mathrm{~Hz},{ }^{3} J_{(H, F)}=18.5 \mathrm{~Hz}, 1 \mathrm{H}, \mathrm{CH}{ }_{2} \mathrm{CF}\right)$, $3.47\left(\mathrm{dd},{ }^{2} J_{(H, H)}=18.2 \mathrm{~Hz},{ }^{3} J_{(H, F)}=21.8 \mathrm{~Hz}, 1 \mathrm{H}, \mathrm{CH} \mathrm{CF}_{2}\right), 1.45\left(\mathrm{~s}, 9 \mathrm{H}, \mathrm{OC}\left(\mathrm{CH}_{3}\right)_{3}\right) ;{ }^{19} \mathrm{~F}-\mathrm{NMR}(235 \mathrm{MHz}$ $\left.[\mathrm{D}] \mathrm{CDCl}_{3}, 298 \mathrm{~K}, \mathrm{TMS}\right) \delta=-63.2\left(\mathrm{~s}, 3 \mathrm{~F}, \mathrm{CF}_{3}\right),-163.9 \mathrm{pm}(\mathrm{s}, 1 \mathrm{~F}, \mathrm{CF}) ;{ }^{13} \mathrm{C}-\mathrm{NMR}\left(91 \mathrm{MHz}[\mathrm{D}] \mathrm{CDCl}_{3}\right.$, $298 \mathrm{~K}, \mathrm{TMS}) \delta=194.7\left(\mathrm{~d},{ }^{2} J_{(C, F)}=18.6 \mathrm{~Hz}\right), 165.6\left(\mathrm{~d},{ }^{2} J_{(C, F)}=27.5 \mathrm{~Hz}\right), 154.7\left(\mathrm{~d},{ }^{3} J_{(C, F)}=3.6 \mathrm{~Hz}\right), 133.9$ 
(s), $132.8\left(\mathrm{q},{ }^{4} J_{(C, F)}=3.2 \mathrm{~Hz}\right), 131.4\left(\mathrm{q},{ }^{2} J_{(C, F)}=33.5 \mathrm{~Hz}\right), 127.4(\mathrm{~s}), 123.4\left(\mathrm{q},{ }^{1} J_{(C, F)}=272.5 \mathrm{~Hz}\right), 122.5$ $\left(\mathrm{q},{ }^{3} J_{(C, F)}=4.0 \mathrm{~Hz}\right), 94.2\left(\mathrm{~d},{ }^{1} J_{(C, F)}=203.5 \mathrm{~Hz}\right), 84.7(\mathrm{~s}), 38.3\left(\mathrm{~d},{ }^{2} J_{(C, F)}=24.2 \mathrm{~Hz}\right), 27.2(\mathrm{~s})$ ppm; HPLC: Daicel Chiralpack AD-H, Hexane $/{ }^{i} \mathrm{PrOH}=99.9: 0.10 .7 \mathrm{~mL} / \mathrm{min}, 254 \mathrm{~nm}, \mathrm{t}_{R}$ (minor) $=20.2 \mathrm{~min}, \mathrm{t}_{S}$ (major) $=23.1 \mathrm{~min}(81 \% e e) ;[\alpha]_{20} \mathrm{D}:-5.6\left(\mathrm{c}=6.2, \mathrm{CHCl}_{3}\right)$. HRMS (ESI): $(\mathrm{M}+\mathrm{Na})^{+}$Calcd for $\mathrm{C}_{15} \mathrm{H}_{14} \mathrm{O}_{3} \mathrm{~F}_{4} \mathrm{Na}$ 341.0771; found 341.0772.

Tert-butyl (R)-2,5,7-trifluoro-1-oxo-2,3-dihydro-1H-indene-2-carboxylate (10i): Following the general procedure, using $\mathrm{Eu}(\mathrm{OTf})_{3}$ and $(S, R)$-ind-pybox, $45 \mathrm{mg}(0.15 \mathrm{mmol}, 71 \%$ yield $)$ of 10i were obtained as a colorless oil from $0.22 \mathrm{mmol}$ of $2 \mathbf{i}$. It was purified by column chromatography on silica-gel (hexane/AcOEt 9:1). IR (ATR) $v=2921,1751$ (C=O), 1721, 1484, 1298, 1149,1040 cm ${ }^{-1} ;{ }^{1} \mathrm{H}-\mathrm{NMR}$ $\left(400 \mathrm{MHz}[\mathrm{D}] \mathrm{CDCl}_{3}, 298 \mathrm{~K}, \mathrm{TMS}\right) \delta=7.01\left(\mathrm{~d},{ }^{3} J_{(H, F)}=6.5 \mathrm{~Hz}, 1 \mathrm{H}, \mathrm{ArH}\right), 6.86\left(\mathrm{t},{ }^{3} J_{(H, F)}=8.2 \mathrm{~Hz}, 1 \mathrm{H}\right.$, $\mathrm{ArH}), 3.74\left(\mathrm{dd},{ }^{2} J_{(H, H)}=10.0,{ }^{3} J_{(H, F)}=17.6 \mathrm{~Hz}, 1 \mathrm{H}\right), 3.42\left(\mathrm{dd},{ }^{2} J_{(H, H)}=15.5,{ }^{3} J_{(H, F)}=23.9 \mathrm{~Hz}, 1 \mathrm{H}\right)$, $\left.1.48 \mathrm{ppm}\left(\mathrm{s}, 9 \mathrm{H}, \mathrm{OC}\left(\mathrm{CH}_{3}\right)_{3}\right) ;{ }^{19} \mathrm{~F}-\mathrm{NMR}\left(376 \mathrm{MHz}^{\mathrm{DD}}\right] \mathrm{CDCl}_{3}, 298 \mathrm{~K}, \mathrm{TMS}\right) \delta=-94.9\left(\mathrm{~d},{ }^{4} J_{(F, F)}=14.7 \mathrm{~Hz}\right.$, $1 \mathrm{~F}, \mathrm{ArF}),-106.4\left(\mathrm{~d},{ }^{4} J_{(F, F)}=14.7 \mathrm{~Hz}, 1 \mathrm{~F}, \mathrm{Ar} F\right),-162.5 \mathrm{ppm}(\mathrm{s}, 1 \mathrm{~F}, \mathrm{CF}) ;{ }^{13} \mathrm{C}-\mathrm{NMR}\left(101 \mathrm{MHz}[\mathrm{D}] \mathrm{CDCl} \mathrm{C}_{3}\right.$, $298 \mathrm{~K}, \mathrm{TMS}) \delta=193.2\left(\mathrm{~d},{ }^{2} J_{(C, F)}=18.2 \mathrm{~Hz}\right), 167.8\left(\mathrm{dd},{ }^{3} J_{(C, F)}=3.9 \mathrm{~Hz},{ }^{1} J_{(C, F)}=259.5 \mathrm{~Hz}\right), 165.8\left(\mathrm{~d},{ }^{2} J_{(C, F)}\right.$ $=24.5 \mathrm{~Hz}), 160.0\left(\mathrm{dd},{ }^{3} J_{(C, F)}=14.2 \mathrm{~Hz},{ }^{1} J_{(C, F)}=267.5 \mathrm{~Hz}\right), 153.4(\mathrm{~m}), 120.1,109.8\left(\mathrm{dd},{ }^{4} J_{(C, F)}=4.2 \mathrm{~Hz}\right.$, $\left.{ }^{2} J_{(C, F)}=22.5 \mathrm{~Hz}\right), 95.4\left(\mathrm{~d},{ }^{1} J_{(C, F)}=202.4 \mathrm{~Hz}\right), 82.5(\mathrm{~s}), 38.9\left(\mathrm{~d},{ }^{2} J_{(C, F)}=24.1 \mathrm{~Hz}\right), 27.8(\mathrm{~s})$ ppm; HPLC: Daicel Chiralpack AD-H, Hexane $/{ }^{i} \mathrm{PrOH}=97: 3,1.0 \mathrm{~mL} / \mathrm{min}, 254 \mathrm{~nm}, \mathrm{t}_{r}$ (major) = $8.7 \mathrm{~min}, \mathrm{t}_{r}($ minor $)$ $=12.9 \min (84 \% e e) ;[\alpha]_{20}{ }^{\mathrm{D}}:-10.7\left(\mathrm{c}=6.8, \mathrm{CHCl}_{3}\right)$. HRMS (ESI): $(\mathrm{M}+\mathrm{Na})^{+}$Calcd for $\mathrm{C}_{14} \mathrm{H}_{13} \mathrm{~F}_{3} \mathrm{O}_{3} \mathrm{Na}$ 309.0709; found 309.0709.

Tert-butyl (R)-2-fluoro-7-bromo-1-oxo-2,3-dihydro-1H-indene-2-carboxylate (10j): Following the general procedure, using $\mathrm{Eu}(\mathrm{OTf})_{3}$ and $(S, R)$-ind-pybox, $48 \mathrm{mg}(0.14 \mathrm{mmol}, 73 \%$ yield $)$ of $\mathbf{1 0 j}$ were obtained as a colorless oil from $0.20 \mathrm{mmol}$ of $\mathbf{2 j}$. It was purified by column chromatography on silica-gel (hexane/AcOEt 9:1). ${ }^{1} \mathrm{H}-\mathrm{NMR}\left(360 \mathrm{MHz}[\mathrm{D}] \mathrm{CDCl}_{3}, 298 \mathrm{~K}, \mathrm{TMS}\right) \delta=7.61\left(\mathrm{~d},{ }^{3} J_{(\mathrm{H}, H)}=7.6 \mathrm{~Hz}, 1 \mathrm{H}\right.$, $\operatorname{ArH}), 7.51\left(\mathrm{t},{ }^{3} J_{(H, H)}=7.6 \mathrm{~Hz}, 1 \mathrm{H}, \mathrm{ArH}\right), 7.45\left(\mathrm{~d},{ }^{3} J_{(H, H)}=7.6 \mathrm{~Hz}, 1 \mathrm{H}, \operatorname{ArH}\right), 3.69\left(\mathrm{dd},{ }^{2} J_{(H, H)}=11.2\right.$, $\left.{ }^{3} J_{(H, F)}=17.6 \mathrm{~Hz}, 1 \mathrm{H}\right), 3.37\left(\mathrm{dd},{ }^{2} J_{(H, H)}=17.6,{ }^{3} J_{(H, F)}=22.9 \mathrm{~Hz}, 1 \mathrm{H}\right), 1.44 \mathrm{ppm}\left(\mathrm{s}, 9 \mathrm{H}, \mathrm{OC}\left(\mathrm{CH}_{3}\right)_{3}\right)$; ${ }^{19} \mathrm{~F}-\mathrm{NMR}\left(235 \mathrm{MHz}[\mathrm{D}] \mathrm{CDCl}_{3}, 298 \mathrm{~K}, \mathrm{TMS}\right) \delta=-163.8 \mathrm{ppm}(\mathrm{s}, 1 \mathrm{~F}) ;{ }^{13} \mathrm{C}-\mathrm{NMR}\left(91 \mathrm{MHz}[\mathrm{D}] \mathrm{CDCl}_{3}, 298\right.$ $\mathrm{K}, \mathrm{TMS}) \delta=193.2\left(\mathrm{~d},{ }^{2} J_{(C, F)}=19.2 \mathrm{~Hz}\right), 165.8\left(\mathrm{~d},{ }^{2} J_{(C, F)}=27.6 \mathrm{~Hz}\right), 153.4\left(\mathrm{~d},{ }^{3} J_{(C, F)}=3.6 \mathrm{~Hz}\right), 136.7(\mathrm{~s})$, $133.4(\mathrm{~s}), 131.3(\mathrm{~s}), 125.4(\mathrm{~s}), 121.2(\mathrm{~s}), 94.5\left(\mathrm{~d},{ }^{1} J_{(C, F)}=202.4 \mathrm{~Hz}\right), 84.5(\mathrm{~s}), 37.4\left(\mathrm{~d},{ }^{2} J_{(C, F)}=24.1 \mathrm{~Hz}\right), 27.8(\mathrm{~s})$ ppm; HPLC: Daicel Chiralpack AD-H, Hexane $/{ }^{i} \mathrm{PrOH}=97: 3,1.0 \mathrm{~mL} / \mathrm{min}, 254 \mathrm{~nm}, \mathrm{t}_{r}(\mathrm{major})=12.1 \mathrm{~min}$, $\mathrm{t}_{r}$ (minor) $=18.9 \min (85 \% e e) ;[\alpha]_{20}{ }^{\mathrm{D}}:-34.7\left(\mathrm{c}=6.3, \mathrm{CHCl}_{3}\right)$. HRMS (ESI): $(\mathrm{M}+\mathrm{Na})^{+}$Calcd for $\mathrm{C}_{14} \mathrm{H}_{14} \mathrm{BrFO}_{3} \mathrm{Na} 351.0003$; found 351.0005.

\section{Conclusions}

In conclusion, we have established a catalytic method for the highly enantioselective $\alpha$-fluorination of a series of alkyl 1-oxo-indanecarboxylates, using europium (III) triflate and $(S, R)$-ind-pybox as pre-catalyst, and NFSI as electrophilic fluorinating agent in acetonitrile at $-30{ }^{\circ} \mathrm{C}$. Selectivities up to $96 \%$ ee could be obtained in mild conditions, and using a commercially available chiral ligand. Results reveal a dependence of the enantiocontrol on the sterically hindrance of the ester in substrates. Access to both enantiomers of the $\alpha$-fluorinated oxo ester is guaranteed by the commercial availability of both $(R, S)$ and $(S, R)$ ind-pybox C2-symmetric ligands.

Supplementary Materials: The following are available online: synthesis and characterization of starting materials 1a-j and 2a-j, and pybox ligands 4 and 5. Spectra, HPLC and CD of compounds 10a-j.

Author Contributions: Conceptualization, A.V.; methodology, A.G. and P.S.; formal analysis, A.G. and P.S.; investigation, A.G. and P.S.; resources, A.V.; data curation, A.G.; writing-original draft preparation, A.V. and A.G.; writing-review and editing, A.V.; supervision, A.V. and A.G.; funding acquisition, A.V.

Funding: This research was funded by Spain's MICINN (CTQ2014-53662-P) and MEC (CTQ2016-81797-REDC) and DURSI -Generalitat de Catalunya (2017 SGR 465).

Acknowledgments: We are thankful for financial support from Spain's MICINN (CTQ2014-53662-P) and MEC (CTQ2016-81797-REDC) and DURSI -Generalitat de Catalunya (2017 SGR 465).

Conflicts of Interest: The authors declare no conflict of interest. 


\section{References}

1. Kirsch, P. Modern Fluoroorganic Chemistry; Wiley-VCH: Weinheim, Germany, 2004.

2. Gladysz, J.A.; Curran, D.E.; Horváth, I.T. Handbook of Fluorous Chemistry; Wiley-VCH: Weinheim, Germany, 2004.

3. Liang, T.; Neumann, C.N.; Ritter, T. Introduction of Fluorine and Fluorine-Containing Functional Groups. Angew. Chem. Int. Ed. 2013, 52, 8214-8264. [CrossRef] [PubMed]

4. Smart, B.E. Fluorine Substituent Effects (on Bioactivity). J. Fluor. Chem. 2001, 109, 3-11. [CrossRef]

5. O'Hagan, D. Understanding Organofluorine Chemistry. An Introduction to the C-F Bond. Chem. Soc. Rev. 2008, 37, 308-319. [CrossRef] [PubMed]

6. Isanbor, C.; O'Hagan, D. Fluorine in Medicinal Chemistry: A Review of Anti-cancer Agents. J. Fluor. Chem. 2006, 127, 303-319. [CrossRef]

7. Müller, K.; Faeh, C.; Diederich, F. Fluorine in Pharmaceuticals: Looking Beyond Intuition. Science 2007, 317, 1881-1886. [CrossRef] [PubMed]

8. Purser, S.; Moore, P.R.; Swallow, S.; Gouverneur, V. Fluorine in Medicinal Chemistry. Chem. Soc. Rev. 2008, 37, 320-330. [CrossRef] [PubMed]

9. Ojima, I. Fluorine in Medicinal Chemistry and Chemical Biology; Wiley-Blackwell: Hobpken, NY, USA, 2009.

10. O'Hagan, D. Fluorine in Health Care: Organofluorine Containing Blockbuster Drugs. J. Fluor. Chem. 2010, 131, 1071-1081. [CrossRef]

11. Ilardi, E.A.; Vitaku, E.; Njardarson, J.T. Data-Mining for Sulfur and Fluorine: An Evaluation of Pharmaceuticals To Reveal Opportunities for Drug Design and Discovery. J. Med. Chem. 2014, 57, 2832-2842. [CrossRef]

12. Wang, J.; Sanchez-Roselló, M.; Aceña, J.L.; del Pozo, C.; Sorochinsky, A.E.; Soloshonok, V.A.; Liu, H. Fluorine in Pharmaceutical Industry: Fluorine-Containing Drugs Introduced to the Market in the Last Decade (2001-2011). Chem. Rev. 2014, 114, 2432-2506. [CrossRef]

13. Ghosh, A.; Nakanishi, T. Frontiers of Solvent-free Functional Molecular Liquids. Chem. Comm. 2017, 53, 10344-10357. [CrossRef]

14. Pihko, P. Enantioselective $\alpha$-Fluorination of Carbonyl Compounds: Organocatalysis or Metal Catalysis? Angew. Chem. Int. Ed. 2006, 45, 544-547. [CrossRef]

15. Zhu, Y.; Han, J.; Wang, J.; Shibata, N.; Sodeoka, M.; Soloshonok, V.A.; Coelho, J.A.S.; Toste, F.D. Modern Approaches for Asymmetric Construction of Carbon-Fluorine Quaternary Stereogenic Centers: Synthetic Challenges and Pharmaceutical Needs. Chem. Rev. 2018, 118, 3887-3964. [CrossRef]

16. Shen, X.; Zhang, W.; Zhang, L.; Luo, T.; Wan, X.; Gu, Y.; Hu, J. Enantioselective Synthesis of Cyclopropanes that Contain Fluorinated Tertiary Stereogenic Carbon Centers: A Chiral $\alpha$-Fluoro Carbanion Strategy. Angew. Chem. Int. Ed. 2012, 51, 6966-6970. [CrossRef]

17. Differding, E.; Ofner, H. N-Fluorobenzenesulfonimide: A Practical Reagent for Electrophilic Fluorinations. Synlett 1991, 187-189. [CrossRef]

18. Nyffeler, P.T.; Gonzalez, S.; Burkart, M.D.; Vicent, S.P.; Wong, C.-H. Selectfluor: Mechanistic Insight and Applications. Angew. Chem. Int. Ed. 2005, 44, 192-202. [CrossRef]

19. Hintermann, L.; Togni, A. Catalytic Enantioselective Fluorination of $\beta$-Ketoesters. Angew. Chem. Int. Ed. 2000, 39, 4359-4362. [CrossRef]

20. Hamashima, Y.; Yagi, K.; Takano, H.; Tamás, L.; Sodeoka, M. An Efficient Enantioselective Fluorination of Various $\beta$-Ketoesters Catalyzed by Chiral Palladium Complexes. J. Am. Chem. Soc. 2002, 124, 14530-14531. [CrossRef]

21. Hamashima, Y.; Takano, H.; Hotta, D.; Sodeoka, M. Immobilization and Reuse of Pd Complexes in Ionic Liquid: Efficient Catalytic Asymmetric Fluorination and Michael Reactions with $\beta$-Ketoesters. Org. Lett. 2003, 5, 3225-3228. [CrossRef]

22. Suzuki, T.; Goto, T.; Hamashima, Y.; Sodeoka, M. Enantioselective Fluorination of tert-Butoxycarbonyl Lactones and Lactams Catalyzed by Chiral Pd(II)-Bisphosphine Complexes. J. Org. Chem. 2007, 72, 246-250. [CrossRef]

23. Ibrahim, H.; Togni, A. Enantioselective Halogenation Reactions. Chem. Commun. 2004, 0, 1147-1155. [CrossRef] 
24. Huber, D.P.; Stanek, K.; Togni, A. Consecutive Catalytic Electrophilic Fluorination/Amination of $\beta$-Keto esters: Toward $\alpha$-Fluoro- $\alpha$-amino Acids? Tetrahedron-Asymmetr 2006, 17, 658-664. [CrossRef]

25. Ma, J.-A.; Cahard, D. Copper(II) Triflate-bis(oxazoline)-catalysed Enantioselective Electrophilic Fluorination of $\beta$-Ketoesters. Tetrahedron-Asymmetr 2004, 15, 1007. [CrossRef]

26. Shibata, N.; Ishimaru, T.; Nagai, T.; Kohno, J.; Toru, T. First Enantio-Flexible Fluorination Reaction Using Metal-Bis(oxazoline) Complexes. Synlett 2004, 10, 1703-1706. [CrossRef]

27. Balaraman, K.; Vasanthan, R.; Kesavan, V. Enantioselective Fluorination of $\beta$-Ketoesters using Tartrate Derived Bidentate Bisoxazoline-Cu(II) Complexes. Tetrahedron-Asymmetr 2013, 24, 919-924. [CrossRef]

28. Gu, X.; Zhang, Y.; Xu, Z.-J.; Che, C.-M. Iron(III)-salan complexes catalysed highly enantioselective fluorination and hydroxylation of $\beta$-keto esters and N-Boc oxindoles. Chem. Commun. 2014, 50, 7870-7873. [CrossRef]

29. Wang, Y.; Wang, H.; Jiang, Y.; Zhang, C.; Shao, J.; Xu, D. Fast, solvent-free and highly enantioselective fluorination of $\beta$-keto esters catalyzed by chiral copper complexes in a ball mill. Green Chem. 2017, 19, 1674-1677. [CrossRef]

30. Comelles, J.; Pericas, A.; Moreno-Mañas, M.; Vallribera, A.; Drudis-Solé, G.; Lledós, A.; Parella, T.; Roglans, A.; García-Granda, S.; Roces-Fernández, L. Highly Enantioselective Electrophilic Amination and Michael Addition of Cyclic $\beta$-Ketoesters Induced by Lanthanides and (S,S)-ip-pybox: The Mechanism. J. Org. Chem. 2007, 72, 2077-2087. [CrossRef]

31. Pericas, A.; Shafir, A.; Vallribera, A. Asymmetric Synthesis of 1-Carbidopa Based on a Highly Enantioselective $\alpha$-Amination. Org. Lett. 2013, 15, 1448-1451. [CrossRef]

32. Pericas, A.; Jiménez, R.; Granados, A.; Shafir, A.; Vallribera, A.; Roglans, A.; Molins, E. Lanthanides-pybox: An Excellent Combination for Highly Enantioselective Electrophilic $\alpha$-Amination of Acyclic $\beta$-Keto Esters. Isolation of Ternary Pybox/Ln/ $\beta$-Keto Ester Complexes. ChemistrySelect. 2016, 1, 4305-4312. [CrossRef]

33. Granados, A.; del Olmo, A.; Peccati, F.; Billard, T.; Sodupe, M.; Vallribera, A. Fluorous l-Carbidopa Precursors: Highly Enantioselective Synthesis and Computational Prediction of Bioactivity. J. Org. Chem. 2018, 83, 303-313. [CrossRef]

34. Pericas, A.; Shafir, A.; Vallribera, A. ZnO-Catalyzed Transesterification of. $\beta$-Keto Esters. Tetrahedron 2008, 64, 9258-9263. [CrossRef]

35. Meng, J.-C.; Fokin, V.V.; Finn, M.G. Kinetic resolution by copper-catalyzed azide-alkyne cycloaddition. Tetrahedron Lett. 2005, 46, 4543-4546. [CrossRef]

36. Wang, Y.-F.; Qiu, J.; Kong, D.; Gao, Y.; Lu, F.; Karmarker, P.G.; Chen, F.-X. The direct electrophilic cyanation of $\beta$-keto esters and amides with cyano benziodoxole. Org. Biomol. Chem. 2015, 13, 365-368. [CrossRef]

Sample Availability: Samples of the compounds are not available from the authors.

(C) 2019 by the authors. Licensee MDPI, Basel, Switzerland. This article is an open access article distributed under the terms and conditions of the Creative Commons Attribution (CC BY) license (http:/ / creativecommons.org/licenses/by/4.0/). 